Rev. salud pública. 14 (1): 53-66, 2012

\title{
Prevalencia de citología anormal e inflamación y su asociación con factores de riesgo para neoplasias del cuello uterino en el Cauca, Colombia
}

\author{
The prevalence of abnormal cytology and inflammation and their \\ association with risk factors for uterine cervical neoplasms in \\ Cauca, Colombia
}

Yaliana Tafurt-Cardona, Claudia P. Acosta-Astaiza y Carlos H. Sierra-Torres

Laboratorio de Genética Humana, Departamento de Ciencias Fisiológicas, Facultad de Ciencias de la Salud, Universidad del Cauca, Popayán, Colombia. hsierra@unicauca.edu.co

Recibido 15 Abril 2011/Enviado para Modificación 10 Febrero 2012/Aceptado 23 Febrero 2012

\section{RESUMEN}

Objetivos Establecer la prevalencia del resultado de citología anormal e inflamación y su asociación con factores de riesgo para neoplasias del cuello uterino en mujeres del departamento del Cauca, Colombia.

Metodología Después de la firma voluntaria del consentimiento informado, las mujeres fueron entrevistadas a través de un cuestionario para colectar variables de tipo sociodemográfico y clínico, incluyendo historia reproductiva, actividad sexual, historia de citología y hábito de fumar. Posteriormente, se procedió a la toma de la citología para su análisis y clasificación según el sistema Bethesda 2001. Un total de 1735 mujeres fueron reclutadas para este estudio.

Resultados Acorde con el resultado de citología, 1061 mujeres presentaron citología normal (61\%), 36 citología anormal (2\%) y 638 cambios celulares reactivos asociados a inflamación (37\%). Los resultados indican que tener relaciones sexuales a temprana edad, la multiparidad, el uso de anticonceptivos hormonales y no realizarse la citología anualmente fueron factores de riesgo asociados a citología anormal.

Conclusiones Estos resultados brindan información valiosa a las instituciones de salud pública para desarrollar mejores programas de cribado para la prevención de neoplasias del cuello uterino en mujeres de la región y el país.

Palabras Clave: Citología, factores de riesgo, papilomavirus humano, inflamación, neoplasias del cuello uterino, Colombia (fuente: DeCS, BIREME).

\section{ABSTRACT}

Objectives Establishing the prevalence of abnormal and inflammation cytology reports and its association with risks factors for uterine cervical neoplasms amongst 
females from the Cauca department in Colombia.

Methodology After signing a consent-form, females were interviewed using a questionnaire to collect socio-demographic and clinical data, including reproductive history, sexual activity, cytology history and smoking habits. Cytology was then taken for analysis and classification according to the 2001 Bethesda System. A total of 1,735 females were recruited for the study.

Results According to the cytology report, 1061 women had normal cytology (61 $\%), 36$ abnormal cytology (2\%) and 638 reactive cellular changes associated with inflammation (37\%). The results indicated that having sexual intercourse at an early age, multiparity, using hormonal contraceptives and not having annual cytology screening were associated with abnormal cytology reports.

Conclusions These results provided valuable information for public health institutions for developing better screening programmes to prevent risks of uterine cervical neoplasms amongst females from our region and throughout Colombia.

Key Words: Cytology, risk factor, human papillomavirus, inflammation, uterine cervical neoplasm, Colombia (source: MeSH, NLM).

nivel mundial el cáncer de cuello uterino (CCU) es el tercer tipo de
cáncer más común en la mujer; en el 2008 se registraron 275000
muertes de las cuales $88 \%$ ocurrieron en países en desarrollo (1). El CCU es responsable de aproximadamente el $2 \%$ del total ponderado de años de vidas perdidas en mujeres jóvenes y de mediana edad $(2,3)$. Según la Agencia Internacional para la Investigación del Cáncer (IARC), en el año 2002 se estimo un total de 86134 nuevos casos y 38238 muertes por CCU en América Latina y el Caribe, siendo la causa más importante de mortalidad en la mujer, más que la tuberculosis, la mortalidad materna o el síndrome de inmunodeficiencia adquirida $(4,5)$. Según la Liga Colombiana Contra el Cáncer, cada 3 horas muere una mujer en nuestro país por CCU, siendo la primera causa de mortalidad en mujeres entre los 35 y 64 años de edad; se registra una incidencia de 7000 casos y mueren 3300 mujeres por esta patología anualmente (6). En el año 2008, se diagnosticaron cerca de 800 mil nuevos casos de los cuales el $50 \%$ tuvieron un desenlace fatal (7). Un estudio de mortalidad por cáncer en Colombia encontró una tasa nacional para CCU de 3,7 por 100000 mujeres y para el Departamento del Cauca de 3,3 (8). De acuerdo con la Secretaria de Salud del Cauca en el año 2007 se reportaron 48 muertes, correspondiendo a una tasa de 7,55 por 100000 mujeres muy por encima de la meta de los objetivos de desarrollo del milenio (ODM) del 2015 de 5,5 (7).

Numerosos estudios epidemiológicos han confirmado que el CCU es causado por el Virus del Papiloma Humano (VPH), un factor necesario 
pero no suficiente para el desarrollo del carcinoma cervical (9-11), el cual es transmitido sexualmente e infecta casi al $75 \%$ de la población sexualmente activa en el mundo (12). La prevalencia del VPH es mayor entre los 20 y los 25 años de edad, etapa de mayor actividad sexual. Sin embargo, en mujeres mayores de 30 años la presencia del VPH indica una infección persistente que aumenta el riesgo de desarrollar neoplasias del cuello uterino y cáncer (13). El factor de riesgo más consistente y documentado para la infección por VPH es el número de compañeros sexuales (14). Otros posibles factores de riesgo son el inicio de relaciones sexuales a temprana edad, la multiparidad, consumo de cigarrillo, anticonceptivos orales, estilo de vida y la exposición a carcinógenos ambientales presentes en el humo de leña, los cuales son importantes co-factores de riesgo para esta neoplasia cervical (15). Estudios de seguimiento en mujeres que han adquirido el VPH muestran que la gran mayoría de ellas (80 \%) han eliminado la infección a los 18 meses (16). Sólo una pequeña proporción de infecciones se manifiesta clínicamente y resulta en lesiones precancerosas, cáncer o lesiones benignas. Por lo tanto, existen otros factores necesarios para la progresión de la carcinogénesis en el cuello uterino.

Laasociación etiológica entre elVPHy elCCU introdujonuevas perspectivas en el campo del diagnóstico, tratamiento y prevención de este cáncer. Se trata de los programas de cribado que convirtieron la citología en una herramienta fundamental para la detección temprana de lesiones precancerosas (17). El cribado masivo como método de detección temprana ha permitido en los países desarrollados disminuir la incidencia, frecuencia y mortalidad de CCU en un $75 \%$, aproximadamente (18). Estos programas requieren la identificación de la población a riesgo en bases de datos, repetición de la citología a cortos intervalos de tiempo, altas tasas de cobertura, controles de calidad en la toma y lectura de la citología, seguimiento estricto de las mujeres con citología anormal, acceso fácil e inmediato a la colposcopia y biopsia ofreciendo un tratamiento adecuado y oportuno $(19,20)$. Sin embargo, el impacto ha sido menor en los países en desarrollo (21). Esta menor efectividad de cribado ha sido asociada a la baja sensibilidad de la citología, puesto que aún en excelentes condiciones de calidad, esta sólo alcanza el $60 \%$ de sensibilidad para neoplasia intraepitelial cervical II (22). Otra de las limitaciones son los falsos negativos, cerca de un tercio de ellos atribuibles a errores en su interpretación y dos tercios a fallas en la toma de muestra y preparación de la placa (23). Por otra parte, los actuales sistemas de salud no permiten la articulación de todos los niveles de atención para la evaluación de los programas de detección y tratamiento (24). 
Previo a la realización de esta investigación, no se encontraron publicaciones acerca de las características de las mujeres que presentan anomalías e inflamación en el resultado de citología en el departamento del Cauca. Ésta información es necesaria tanto para instituciones públicas como para entes encargados de planificar políticas de salud. Por tal motivo, este estudio constituye el primer informe para esta población, con el objetivo de establecer la prevalencia del resultado de citología anormal e inflamación y su asociación con factores de riesgo para neoplasias del cuello uterino.

\section{MATERIALES Y MÉTODOS}

Población de estudio y muestreo

Este estudio observacional, de corte transversal, se realizó en mujeres mayores de 18 años de edad, que asistieron a los servicios de citología en el Hospital Universitario San José y la Unidad de Salud de la Universidad del Cauca en la ciudad de Popayán, entre septiembre 2007 y agosto de 2008. La población objeto de estudio fue definida como mujeres con un cuello uterino sano y con historia de resultado de citología "normal" en el último año previo al ingreso en el estudio. Los criterios de exclusión fueron exposición a drogas quimio-terapéuticas, historia previa de tratamiento por infección con VPH (Ej. Crioterapia, conización), histerectomía, negación a participar en el estudio o que su estado de salud no se lo permitiera. Después de la firma voluntaria del consentimiento informado, según lo dispuesto en la Resolución No. 8430 de 1993 del Ministerio de Salud, las participantes fueron entrevistadas a través de un cuestionario para colectar las variables de tipo sociodemográfico y clínico, incluyendo historia reproductiva, actividad sexual, historia de citología y hábito de fumar. Después de la entrevista, se procedió a la toma de la muestra exocervical con espátula de Ayre y endocervical, utilizando un citocepillo. Posteriormente, se remitió la muestra para la interpretación citológica de acuerdo a la nomenclatura del Sistema de Bethesda 2001 (26). La encuesta y toma de citología fueron realizadas por un auxiliar de enfermería o enfermera profesional debidamente entrenada. Todos los procedimientos y protocolos utilizados en el estudio fueron revisados y aprobados por el Comité de Ética para la Investigación Científica de la Universidad del Cauca, adoptando los principios bioéticos establecidos en la Declaración de Helsinki (25). Los cuestionarios y las muestras fueron codificados para proteger la confidencialidad de las participantes. 
Análisis estadístico

El análisis de datos se realizó con el paquete estadístico SPSS para Windows, versión 10 (SPSS Inc., Chicago, IL, EUA). Las variables continuas se expresaron con la media \pm desviación estándar y las variables discretas se expresaron en frecuencias y proporciones. Adicionalmente, se realizó un análisis estadístico mediante la prueba de ANOVA para la comparación de medias y de chi-cuadrado $\left(x^{2}\right)$ para la comparación de proporciones.

\section{RESULTADOS}

Un total de 1735 mujeres fueron reclutadas para este estudio. La Tabla 1 indica las características sociodemográficas de la población. La edad promedio de la población fue $36,1 \pm 11,5$. El $32 \%$ de las mujeres (554/1 735) se encontraron en edades entre los 31 y 40 años. La mayoría de la población procedía del área urbana (66\%), especialmente del centro del Cauca (84 $\%$ ). El $32 \%$ de las mujeres reportaron un nivel educativo bajo ( $\leq$ primaria). En cuanto al estado civil, el $57 \%$ de las mujeres indicaron estar casadas. Solamente 158 mujeres reportaron el consumo de cigarrillo, el $91 \%$ restante de la población no acostumbraba fumar.

Como se indica en la Tabla 2, la población de estudio fue discriminada de acuerdo al resultado de citología, en la cual se incluyó un total de 1061 mujeres con resultado de citología normal (61 \%), 36 mujeres con citología anormal (2\%) y 638 mujeres con resultado de cambios celulares reactivos asociados a inflamación (37\%). Entre las mujeres con inflamación, 26,3 $\%$ presentaron infecciones correspondientes a Gardanella vaginalis $(24,6$ $\%)$, Trichomona vaginallis $(0,8 \%)$ y Candida albicans (0,9\%) - datos no mostrados. Se observo que el $86 \%$ de las mujeres con citología anormal inicio relaciones sexuales a temprana edad $(\mathrm{p}=0.007)$ y el $44 \%$ de ellas habían tenido más de un compañero sexual ( $\mathrm{p}=0.078)$. 
Tabla 1. Características socio demográficas de la población estudio

\begin{tabular}{|c|c|}
\hline Características & Población (\%) \\
\hline Total & 1735 \\
\hline \multicolumn{2}{|l|}{ Edad (años) } \\
\hline Media \pm DS & $36,13 \pm 11,51$ \\
\hline$<=20$ & $110(6)$ \\
\hline $21-30$ & $554(32)$ \\
\hline $31-40$ & $469(27)$ \\
\hline $41-50$ & $404(23)$ \\
\hline $51-60$ & $153(9)$ \\
\hline $61-70$ & $39(2)$ \\
\hline$>70$ & $6(1)$ \\
\hline \multicolumn{2}{|l|}{ Procedencia } \\
\hline Rural & $587(34)$ \\
\hline Urbano & $1148(66)$ \\
\hline \multicolumn{2}{|c|}{ Zona de procedencia } \\
\hline Centro & $1470(84)$ \\
\hline Norte & $7(1)$ \\
\hline Sur & $82(4)$ \\
\hline Oriente & $8(1)$ \\
\hline Occidente & $2(1)$ \\
\hline Bota caucana & $4(1)$ \\
\hline Macizo & $47(2)$ \\
\hline Otros & $115(6)$ \\
\hline \multicolumn{2}{|l|}{ Nivel educativo } \\
\hline Ninguno & $69(4)$ \\
\hline Primaria & $485(28)$ \\
\hline Secundaria & $572(33)$ \\
\hline Técnico & $233(13)$ \\
\hline Universitario & $311(18)$ \\
\hline Otros & $65(4)$ \\
\hline \multicolumn{2}{|l|}{ Estado civil } \\
\hline Soltera & $580(33)$ \\
\hline Casada & $990(57)$ \\
\hline Divorciada & $116(7)$ \\
\hline Viuda & $49(3)$ \\
\hline \multicolumn{2}{|l|}{ Fumar } \\
\hline $\mathrm{Si}$ & $158(9)$ \\
\hline No & $1577(91)$ \\
\hline
\end{tabular}


Tabla 2. Algunos factores de riesgo para CCU y su asociación con el resultado de citología

\begin{tabular}{|c|c|c|c|c|}
\hline Características & $\begin{array}{c}\text { C. normal } \\
n(\%)\end{array}$ & $\begin{array}{c}\text { C. anormal } \\
n(\%)\end{array}$ & $\begin{array}{c}\text { Inflamación } \\
\mathrm{n}(\%)\end{array}$ & $\mathrm{P}$ \\
\hline Total & $1061(61)$ & $36(2)$ & $638(37)$ & N.A \\
\hline \multicolumn{5}{|l|}{ Edad primera relación sexual } \\
\hline Media \pm DS & $18,38 \pm 3,5$ & $16,69 \pm 1,81$ & $17,97 \pm 3,16$ & $0,002^{a}$ \\
\hline$\leq 18$ & $670(63)$ & $31(86)$ & $428(67)$ & \\
\hline$>18$ & $391(37)$ & $5(14)$ & $210(33)$ & $0,007^{b}$ \\
\hline \multicolumn{5}{|c|}{ Numero de compañeros sexuales } \\
\hline$\leq 2$ & $669(63)$ & $20(56)$ & $432(68)$ & \\
\hline$>2$ & $392(37)$ & $16(44)$ & $206(32)$ & $0,078^{b}$ \\
\hline \multicolumn{5}{|l|}{ Paridad } \\
\hline$\leq 2$ & $797(75)$ & $22(61)$ & $397(62)$ & \\
\hline$>2$ & $264(25)$ & $18(39)$ & $241(38)$ & $0,001^{b}$ \\
\hline \multicolumn{5}{|c|}{ Su compañero tuvo relaciones antes de ser su pareja } \\
\hline $\mathrm{Si}$ & $770(72)$ & $24(67)$ & $470(74)$ & \\
\hline No & $103(10)$ & $4(11)$ & $66(10)$ & \\
\hline No sabe & $188(18)$ & $8(22)$ & $102(16)$ & $0,789^{b}$ \\
\hline \multicolumn{5}{|c|}{ Su compañero se relacionó con prostitutas } \\
\hline $\mathrm{Si}$ & $142(13)$ & $4(11)$ & 78 (13) & \\
\hline No & $395(37)$ & $15(42)$ & $240(37)$ & \\
\hline No sabe & $524(50)$ & $17(47)$ & $320(50)$ & $0,940^{b}$ \\
\hline \multicolumn{5}{|c|}{ Su compañero le ha sido infiel } \\
\hline $\mathrm{Si}$ & $455(43)$ & $12(33)$ & $295(46)$ & \\
\hline No & $226(21)$ & $10(28)$ & $119(19)$ & \\
\hline No sabe & $380(36)$ & $14(39)$ & $224(35)$ & $0,700^{b}$ \\
\hline \multicolumn{5}{|l|}{ Uso de condón } \\
\hline Siempre & $66(6)$ & $4(11)$ & $30(5)$ & \\
\hline A veces & $539(51)$ & $18(50)$ & $255(40)$ & \\
\hline Nunca & $456(43)$ & $14(39)$ & $353(55)$ & $0,001^{b}$ \\
\hline \multicolumn{5}{|c|}{ Uso de Anticonceptivos hormonales } \\
\hline $\mathrm{Si}$ & $495(47)$ & $22(61)$ & $284(44)$ & \\
\hline No & $566(53)$ & $14(39)$ & $354(55)$ & $0,133^{b}$ \\
\hline \multicolumn{5}{|c|}{ Tiempo de Uso de Ant. hormonales } \\
\hline Media \pm DS & $3,47 \pm 3,76$ & $3,31 \pm 4,10$ & $3,56 \pm 4,37$ & $0,943^{a}$ \\
\hline$\leq 4$ & $354(71)$ & $14(64)$ & $205(72)$ & \\
\hline$>4$ & $141(29)$ & $8(36)$ & $79 \quad(28)$ & $0,693^{b}$ \\
\hline \multicolumn{5}{|l|}{ Conocimiento de Citología } \\
\hline $\mathrm{Si}$ & $916(86)$ & $32(89)$ & $521(82)$ & \\
\hline No & $145(14)$ & $4(11)$ & $117(18)$ & $0,027^{b}$ \\
\hline \multicolumn{5}{|l|}{ Frecuencia de Citología } \\
\hline Nunca & $119(11)$ & $8(22)$ & $78(12)$ & \\
\hline Alguna vez & 942 (89) & $28(78)$ & $561(88)$ & $0,125^{b}$ \\
\hline 1 vez al año & $583(55)$ & $16(44)$ & $304(48)$ & \\
\hline 1 vez cada $2-3$ años & $230(22)$ & $6(17)$ & $156(25)$ & \\
\hline 1 vez cada $4-5$ años & 88 (8) & $4(11)$ & $61(9)$ & \\
\hline 1 vez cada 6-10 años & $41 \quad(4)$ & $2(6)$ & $39 \quad(6)$ & $0,052^{b}$ \\
\hline
\end{tabular}


El $39 \%$ de mujeres con citología anormal reportaron ser multíparas, comparado con el $25 \%$ y $38 \%$ de mujeres con citología normal e inflamación, indicando una diferencia estadísticamente significativa entre los grupos $(p=0.001)$. Respecto a las variables de comportamiento sexual del compañero, no se observaron diferencias significativas.

El uso de anticonceptivos hormonales fue mayor en las mujeres con resultado de citología anormal (61\%) en comparación con citología normal $(47 \%)$ e inflamación (44\%). El $36 \%$ de mujeres con citología anormal reportaron un uso mayor de 4 años, respecto a mujeres con citología normal (29\%) e inflamación (28\%). Los anticonceptivos más frecuentemente usados fueron el dispositivo intrauterino (DIU), la píldora, la inyección y el condón. Sin embargo, el método de barrera (condón) fue usado en un $45 \%$ de las mujeres con citología con inflamación, con respeto al $57 \%$ y $61 \%$ de mujeres con citología normal y anormal.

A pesar que más del $80 \%$ de la población refirió conocer acerca de la citología, el $22 \%$ de las mujeres con citología anormal nunca se había realizado la citología y entre aquellas que indicaron realizarse la citología (78 \%), menos de la mitad se la realizaban anualmente (44\%).

\section{DISCUSIÓN}

Este estudio de prevalencia presenta factores de riesgo para CCU asociados a inflamación y anormalidades en los resultados de citología, en una población del departamento del Cauca. La población de estudio fue discriminada de acuerdo al resultado de citología, en donde se obtuvo citologías normales, anormales y cambios celulares reactivos asociados a inflamación. Se caracterizo por ser mujeres, en su mayoría, procedentes de áreas urbanas y con un nivel educativo bajo ( $\leq$ primaria), lo cual coincide con estudios de prevalencia realizados en Bogotá (36 \%) y Medellín (26,7 \%), en donde la población también presentó un nivel educativo bajo $(24,27)$. El nivel educativo bajo ha sido previamente descrito en la literatura como factor de riesgo para el desarrollo de lesiones intraepiteliales escamosas de alto grado $(28,29)$. Estos resultados, indican la importancia que tiene la educación en estimular a la mujer para adoptar estilos de vida saludables que reduzcan el riesgo de esta patología.

Estudios previos demuestran que el éxito de los programas de detección temprana, radican en el reconocimiento de la población en riesgo a desarrollar 
$\mathrm{CCU}$, realizando un seguimiento completo de mujeres con resultado de citología anormal (30). Este estudio identifico mujeres con resultado de citología anormal y cambios celulares reactivos asociados a inflamación en donde el 1/4 de la población presentó infección vaginal por Vaginosis bacteriana. Varios estudios indican que la citología inflamatoria puede estar asociada a procesos inespecíficos del cérvix, o secundarios a infecciones del tracto genital inferior, por C. trachomatis, Candida Albicans, Tricomonas vaginalis, vaginosis bacteriana, virus Herpes simplex o a la presencia del dispositivo intrauterino (31-34). La citología inflamatoria también puede estar relacionada a la presencia del VPH, donde la respuesta inmune en el huésped promueve el reclutamiento de células inflamatorias $(35,36)$. Se ha descrito que la infección por VPH puede afectar cerca del $10 \%$ de las mujeres sin alteraciones en la citología (37). Sin embargo, la utilidad de la citología inflamatoria como predictor de lesión premaligna para CCU, ha sido rechazada por algunos autores $(38,39)$, otros consideran que la citología inflamatoria persistente debe ir acompañada de colposcopia, debido a que se encuentran anormalidades en la biopsia desde un $20 \%$ hasta un $70 \%(35,40,41)$.

El inicio de relaciones sexuales a temprana edad aumenta el riesgo de CCU independientemente del número de compañeros sexuales, este ultimo parámetro solo expresa la probabilidad de infección con VPH (9). Estudios epidemiológicos han reportado que el riesgo de neoplasia cervicouterina aumenta en las mujeres que inician relaciones sexuales durante la adolescencia puesto que en esa edad el cuello uterino es particularmente susceptible a las proteínas oncogénicas liberadas por el VPH (10).

Se ha demostrado que el cuello uterino es más vulnerable durante la menarquía, cuando el tejido escamocolumnar sufre metaplasia y una infección con VPH podría alcanzar el tejido basal y establecer una infección persistente (42). En nuestro estudio, el $70 \%$ de las mujeres con citología anormal refirieron haber tenido su primera relación sexual antes de los 18 años, lo cual sugiere que existe un periodo de mayor riesgo durante esta etapa. Estos resultados coinciden con estudios previos para neoplasia cervical realizados en nuestra región (15).

La multiparidad es un factor de riesgo para CCU, debido a que el embarazo provoca un estado de inmunosupresión o el traumatismo mismo producido durante el parto aumentan la susceptibilidad del huésped a la infección por VPH (43). En nuestro estudio el $39 \%$ de mujeres con citología anormal y 38 
$\%$ con inflamación presentan mayor número de partos que las mujeres con resultado de citología normal. Se ha reportado que las mujeres con dos o más hijos tienen un riesgo $80 \%$ mayor con respecto a las nulíparas de presentar una lesión intraepitelial $(44,45)$. Las variables asociadas al comportamiento sexual del compañero, en cuanto a relaciones sexuales antes de ser su pareja, con prostitutas o si le ha sido infiel, no muestran diferencias estadísticamente significativas entre el resultado de citología normal, anormal e inflamación. Esto podría explicarse por la relativa igualdad en el comportamiento de riesgo presentada en los tres grupos de resultados citológicos. Sin embargo, se sugiere que una mujer puede correr mayor riesgo debido al comportamiento de su compañero sexual, donde la historia sexual de él podría ser tan importante como la propia (9). Un estudio realizado en Brasil encontró que los compañeros sexuales de mujeres con CCU habían tenido la primera relación sexual a edad precoz, historia de enfermedades venéreas y visitaban frecuentemente prostitutas (46).

La mayor parte de los estudios epidemiológicos sobre hormonas y CCU aportan evidencia sólida de que en presencia delVPH, el uso de anticonceptivos hormonales durante más de 5 años aumenta el riesgo hasta 4 veces de desarrollar un carcinoma invasor de cérvix, aunque este riesgo disminuye progresivamente con el tiempo desde la interrupción de su uso (47). En el presente estudio, el uso de anticonceptivos hormonales no fue significativo entre los distintos resultados de citología. Sin embargo, la mayoría de la población de estudio reportó usar este tipo de método. El uso de hormonas exógenas, como los anticonceptivos o terapia de reemplazo hormonal pueden influir en las infecciones por VPH de diferentes maneras. Se ha indicado que el genoma del VPH contiene un segmento de reconocimiento hormonal, que podría mostrar un proceso interactivo entre los anticonceptivos orales y el VPH (27). En cuanto al uso de métodos anticonceptivos de barrera, este estudio no reporto significancia entre el usar este método y nunca haberlo usado. Sin embargo, las mujeres con resultado de citología inflamatoria reportaron en un $55 \%$ nunca haber usado este método, el cual es considerado como un factor protector para neoplasia cervical puesto que disminuye el riesgo de transmisión de VPH (48).

Los resultados revelan que prácticamente todas las mujeres en este estudio tienen conocimiento de la citología, estos resultados son similares a los disponibles en la Encuesta Nacional de Factores de Riesgo (ENFREC II), realizada en 1998, en la cual el conocimiento sobre la citología fue 96,1 
\% en mujeres entre 18 y 69 años (49). Sin embargo, nuestros resultados contrastan con algunos estudios realizados en otros países de Latinoamérica como Brasil, en los que $14 \%$ de las mujeres no tenían conocimiento sobre la citología (50). La frecuencia de citología obtenida en este estudio indica que el $17 \%$ de mujeres con citología anormal y el $25 \%$ con inflamación acostumbraban a realizarse la citología 1 vez cada 2 o tres años. Uribe y cols, reportan que en Colombia el 33,9 \% de las mujeres se toman la citología de forma esporádica o nunca lo han hecho y que solo el 34,9\% se tomaron la citología en el último año $(30,38)$. Se ha determinado que al realizar la citología anualmente durante al menos 3 años consecutivos, reduce la tasa de incidencia para carcinoma invasor en un $91,2 \%$ y disminuye la mortalidad en un $60 \%$ para CCU $(21,43)$. Esto no sucede en nuestro medio debido a la dificultad para hacer el seguimiento de las mujeres que se hacen la citología, puesto que muchas de las mujeres que tuvieron un resultado anormal no vuelven a una nueva consulta o para el tratamiento (51). En el departamento del Cauca existe una relación directa entre el porcentaje de mujeres que nunca se han tomado una citología y las altas tasas de mortalidad por CCU (52). Según la encuesta nacional de salud y demografía del 2005 de este departamento, informa que el $74 \%$ de las mujeres entre 18 a 69 años se realizaron una citología vaginal, de las cuales el 7 \% resultaron anormales (7).

En conclusión, el presente estudio da a conocer la prevalencia de algunos factores de riesgo con respecto al resultado de la citología. La frecuencia en la toma de la citología es fundamental puesto que se considera un factor de evidencia disponible, para posibles lesiones precancerosas. Por lo tanto, se sugiere que frente al reporte de citología cervical con inflamación persistente y una vez descartados posibles agentes biológicos, químicos o físicos como responsables de la inflamación, se debe realizar un seguimientos citológico o colposcopia mas biopsia de la población en riesgo, para detectar de manera temprana la progresión a lesiones intraepiteliales escamosas. Además, demuestra la necesidad de fomentar la educación como medio para promover un estilo de vida saludable y el mejoramiento en los programas de cribado. Los datos sugieren que el inicio de relaciones sexuales a temprana edad, la multiparidad, uso de anticonceptivos hormonales y la frecuencia de la citología son importantes co-factores de riesgo para neoplasia cervical. Estos resultados brindan información valiosa a las instituciones de salud pública para desarrollar mejores programas de cribado que brinden promoción y prevención de neoplasia cervical en la región y el país 
Agradecimientos: Y. Tafurt fue financiada por el programa Jóvenes Investigadores e Innovadores de Colciencias, contrato No. 085-2007. Los autores expresan su agradecimiento a la Universidad del Cauca, la Vicerrectoría de Investigaciones y la Facultad de Ciencias de la Salud por su apoyo y colaboración para la ejecución de este proyecto. También agradecen a los profesionales y pacientes del Hospital Universitario San José y la Unidad de Salud de la Universidad del Cauca y al personal del Laboratorio de Genética Humana por su participación y asistencia en el desarrollo de esta investigación.

\section{REFERENCIAS}

1. Ferlay J, Shin HR, Bray F, Forman D, Mathers C, Parkin DM. Estimates of worldwide burden of cancer in 2008: GLOBOCAN 2008. Int J Cancer. 2010; 127:2893-917.

2. Yang BH, Bray FI, Parkin DM, Sellors JW, Zhang ZF. Cervical cancer as a priority for prevention in different world regions: an evaluation using years of life lost. Int J Cancer. 2004; 109:418-24.

3. Franco EL, Duarte Franco E, Ferenczy A. Cervical cancer: epidemiology, prevention and the role of human papillomavirus infection. CMAJ. 2001; 164:1017-25.

4. Pisani P, Bray F, Parkin DM. Estimates of the world wide prevalence of cancer for 25 sites in the adult population. Int J Cancer. 2002; 197:72-81.

5. Muñoz N. The global burden of cervical cancer in Latin America and the Caribbean: perspectives for prevention. Salud Pública Mex. 2007; 49:29-31.

6. Piñeros M, Ferlay J, Murillo R. Incidencia estimada de cáncer en Colombia a nivel departamental y nacional. Salud Pública Mex. 2006; 48:455-65.

7. Secretaria de salud del Cauca. Indicadores de estadisticas año 1998-2007 [Internet]. Disponible en: http://www.saludcauca.gov.co/sistema-integrado-de-informacion/estadisticas.html Consultado en Marzo de 2011.

8. Ochoa-Jaramillo FL, Montoya-Vélez LP. Mortalidad por cáncer en colombia en 2005. Rev Fac Med Unal. 2009; 57:304-315.

9. Bosch FX, Lorincz A, Muñoz N, Meijer CJ, Shah KV. The causal relation between human papillomavirus and cervical cancer. J Clin Pathol. 2002; 55:244-65.

10. Bosch, FX, Munoz N. The viral etiology of cervical cancer. Virus Res. 2002; 89:183-90.

11. Walboomers JM, Jacobs MV, Manos MM, Bosch FX, Kummer JA, Shah KV, Snijders PJ, Peto $J$, Meijer CJ, Muñoz N. Human papillomavirus is a necessary cause of invasive cervical cancer worldwide. J Pathol. 1999; 189:12-9.

12. Burchell AN, Winer RL, de Sanjosé S, Franco EL. Chapter 6: Epidemiology and transmission dynamics of genital HPV infection. Vaccine. 2006; 24:52-61.

13. Blomberg K, Widmark C, Ternestedt BM, Törnberg S, Tishelman C. Between youth and adulthood: focus group discussions with 30-year-old women about cervical cancer and its prevention in urban Sweden. Cancer Nurs. 2011; 34:E11-20.

14. Muñoz N, Méndez F, Posso H, Molano M, van den Brule AJ, Ronderos M, Meijer C, Muñoz A. Incidence, duration, and determinants of cervical human papillomavirus infection in a cohort of Colombia women with normal cytological results. J Infect Dis. 2004; 190: 2077-87.

15. Sierra-Torres $\mathrm{CH}$, Acosta-Aragón MP, Orejuela-Aristizabal L. Papilomavirus y factores asociados a neoplasia intraepitelial cervical de alto grado en Cauca, Colombia. Rev Salud Pública (Bogotá). 2006; 8:47-58.

16. Schiffman M, Kjaer SK. Chapter 2: Natural history of anogenital human papillomavirus infection and neoplasia. J Natl Cancer Inst Monogr. 2003; 31:14-9. 
17. Valdespino VM, Valdespino VE. Cervical cancer screening: state of the art. Curr Opin Obstet Gynecol. 2006; 18:35-40.

18. Cuzick J, Szarewski A, Cubie H, Hulman G, Kitchener H, Luesley D, McGoogan E, Menon U, Teery G, Edwards R, Brooks C, Desai M, Gie C, Ho L, Jacobs I, Pickles C, Sasieni P. Management of women who test positive for high-risk types of human papillomavirus: the Hart study. Lancet. 2003; 362:1871-76.

19. Sherman ME, Lorincz AT, Scott DR, Wacholder S, Castle PE, Glass AG, Mielzynska Lohnas I, Rush BB, Schilffman M. Baseline cytology, human papillomavirus testing, and risk for cervical neoplasia: A 10-year cohort analysis. J Natl Cancer Inst.. 2003; 95:46-52.

20. Sankaranarayanan R, Budukh AM, Rajkumar R. Effective screening programmes for cervical cancer in low- and middle- income developing countries. Bull World Health Organ. 2001; 79:954-961.

21. Miller AB. Quality assurance in screening strategies. Virus Res. 2002; 89:295-99.

22. Kitchener HC, Castle PE, Cox JT. Chapter 7: Achievements and limitations of cervical cytology screening. Vaccine. 2006; 24:63-70.

23. Serman F. Cáncer cervicouterino: epidemiologia, historia natural y rol del virus papiloma humano: perspectivas en prevención y tratamiento. Rev Chil Obstet Ginecol. 2002; 67:318-23.

24. Grisales H, Vanegas AP, Gaviria A, Castaño J, Mora Martín A, Borrero M, Rojas C, Arbeláez MP, Sánchez G. Prevalencia de anormalidades de células epiteliales y factores asociados en mujeres de un municipio rural colombiano. Biomédica. 2008; 28:271-83.

25. World Medical Association. The Helsinki declaration. Orv Hetil 1965; 106:1715-6.

26. Solomon D, Davey D, Kurman R, Moriarty A, O'Connor D, Prey M, Raab S, Sherman M, Wilbur D, Wright T Jr, Young N. The 2001 Bethesda System: terminology for reporting results of cervical cytology. JAMA. 2002; 287:2114-9.

27. Molano M, Posso H, Weiderpass E, van den Brule AJ, Ronderos M, Franceschi S. Meijer CJ, Arslan A, Muñoz N. Prevalence and determinants of HPV infection among Colombian women with normal cytology. Br J Cancer. 2002; 87:324-33.

28. Lazcano Ponce E, Alonso P, Ruiz Moreno JA, Hernández Avila M. Recommendations for cervical cancer screening programs in developing countries. The need for equity and technological development. Salud Pública Mex. 2003; 45:449-62.

29. Capurro I, Rojo JA, Pino T, Vásquez C, Garay J, Venegas M. Programa de detección y control de cáncer de cuello uterino en servicio salud Araucania Sur. Rev Chil Obstet Ginecol. 2002; 67:114-120.

30. Uribe Pérez CJ, Díaz Martínez LA, Ortiz Serrano R, Meza Durán EL. Pap smear prevalence and that of pre-malignant and malignant cervical lesions amongst women living in the Carmen initiative demonstration area, Bucaramanga, Colombia. Rev Colomb Obstet Ginecol. 2006; 57:10-8.

31. Paler RJ Jr, Simpson DR, Kaye AM, Gunn S, Felix JC. The relationship of inflammation in the papanicolaou smear to Chlamydia trachomatis infection in a high-risk population. Contraception. 2000; 61:231-4.

32. Franceschi S, Smith JS, van den Brule A, Herrero R, Arslan A, Anh PT, Bosch FX, Hieu NT, Matos E, Posso H, Qiao YL, Shin HR, Sukvirach S, Thomas JO, Snijders PJ, Muñoz N, Meijer CJ. Cervical infection with Chlamydia trachomatis and Neisseria gonorrhoeae in women from ten areas in four continents. A cross-sectional study. Sex Transm Dis. 2007; 34:563-9.

33. Arora R, Kumar A, Prusty BK, Kailash U, Batra S, Das BC. Prevalence of high-risk human papillomavirus (HR-VPH) types 16 and 18 in healthy women with cytologically negative Pap smear. Eur J Obstet Gynecol Reprod Bio. 2005; 121:104-9.

34. Agarwal K, Sharma U, Acharya V. Microbial and cytopathological study of intrauterine contraceptive device users. Indian J Med Sci. 2004; 58:394-9. 
35. Seckin N, Turhan NO, Ozmen S, Ersan F, Avsar F Ustun H. Routine colposcopic evaluation of patients with persistent inflammatory cellular changes on Pap smear. Int J Gynaecol Obstet .1997; 59:25-9.

36. Boccardo E, Lepique AP, Villa LL. The role of inflammation in HPV carcinogenesis. Carcinogenesis. 2010; 31:1905-12.

37. Rodríguez-Reyes ER, Cerda-Flores RM, Solis-Rios NP, Quiñones-Perez JM, CortésGutiérrez El. Identification and typification of the human papilloma virus in women using the "Timely Detection of Cancer" program in Durango, Mexico. Ginecol Obstet Mex. 2003; 71:471-5.

38. Malik SN, Wilkinson EJ, Drew PA, Hardt NS. Benign cellular changes in Pap smears. Causes and significance. Acta Cytol. 2001; 45:5-8.

39. Mitchell H. Outcome after a cervical cytology report of low-grade squamous abnormality in Australia. Cancer. 2005; 105):185-93.

40. McLachlan N, Patwardhan JR, Ayer B, Pacey NF. Management of suboptimal cytologic smears. Persistent inflammatory smears. Acta Cytol. 1994; 38:531-6.

41. Gaitán Duarte H, Rubio JA, Eslava Schmalbach J. Asociación de la citología cervico-vaginal inflamatoria con la lesión intraepitelial cervical en pacientes de una clínica de salud sexual y reproductiva en Bogotá, Colombia 1999-2003. Rev Salud Pública (Bogotá). 2004; 6:253-269.

42. Sierra-Torres $\mathrm{CH}$, Tyring SK, Au WW. Risk contribution of sexual behavior and cigarette smoking to cervical neoplasia. Int J Gynecol Cancer. 2003; 13:617-25.

43. Rodríguez Salva A, Echavarría Aguilera A, Murlá PA, Vázquez González C. Factores de riesgo del cáncer de cérvix en el municipio del Cerro. Rev Cubana Hig Epidemiol. 1999; 37:40-6.

44. Castañeda IMS, Toledo CR, Aguilera DM. Risk factors for cervico uterine cancer in women in Zacatecas. Salud Publica Mex. 1998; 40:330-8.

45. Ortiz-Serrano R, Uribe C, Diaz LA, Dangond Y. Factores de riesgo para cáncer de cuello uterino. Rev Colomb Obstet Ginecol. 2004; 55:146-160.

46. Schlecht NF, Kulaga S, Robitaille J, FerreiraS, Santos M, Miyamura RA. Persistent human papillomavirus infection as a predictor of cervical intraepithelial neoplasia. JAMA. 2001; 286:3106-14.

47. Moreno V, Bosch FX, Muñoz N, Meijer CJ, Shah KV, Walboomers JM, Herrero R, Franceschi S. Effect of oral contraceptives on risk of cervical cancer in women with human papillomavirus infection: the IARC multicentric case-control study. Lancet. 2002 359:1085-92.

48. Coker AL, Sanders LC, Bond SM, Gerasimova T, Pirisi L. Hormonal and barrier methods of contraception, oncogenic human papillomaviruses, and cervical squamous intraepithelial lesion development. J Womens Health Gend Based Med. 2001; 10:441-9.

49. Ministerio de Salud, Centro Nacional de Consultoría. Conocimientos, actitudes y prácticas en detección temprana de cáncer cérvico-uterino y de mama.II Estudio Nacional de Factores de Riesgo de Enfermedades Crónicas (ENFREC II). Tomo IV. Bogotá D. C.: Ministerio de Salud; 1999.

50. De Quadros CA, Victora CG, da Costa JS. Coverage and focus of a cervical cancer prevention program in southern Brazil. Rev Panam Salud Publica. 2004; 16:223-32.

51. Profamilia. Encuesta nacional de demografia y salud 2005. Bogotá: Profamilia ; 2005.

52. Piñeros M, Cendales R, Murillo R, Wiesner C, Tovar S. Cobertura de la citología de cuello uterino y factores relacionados en Colombia 2005. Rev Salud Pública (Bogotá). 2007; 9:327-341. 